\title{
Minority-spin impurity band in n-type (In,Fe)As: A materials perspective for ferromagnetic semiconductors
}

\author{
Masaki Kobayashi $\odot, 1,2,3,4,{ }^{*}$ Le Duc Anh, ${ }^{4,5,6}$ Jan Minár, ${ }^{7}$ Walayat Khan, ${ }^{8}$ Stephan Borek, ${ }^{9}$ Pham Nam Hai, ${ }^{3,4,10}$ \\ Yoshihisa Harada $\odot,{ }^{11}$ Thorsten Schmitt, ${ }^{1}$ Masaharu Oshima, ${ }^{2}$ Atsushi Fujimori $\odot,{ }^{12,13}$ \\ Masaaki Tanaka $\mathbb{0}^{3,4}$ and Vladimir N. Strocov (1) ${ }^{1}$ \\ ${ }^{1}$ Swiss Light Source, Paul Scherrer Institute, CH-5232 Villigen PSI, Switzerland \\ ${ }^{2}$ Department of Applied Chemistry, School of Engineering, The University of Tokyo, 7-3-1 Hongo, Bunkyo-ku, Tokyo 113-8656, Japan \\ ${ }^{3}$ Department of Electrical Engineering and Information Systems, The University of Tokyo, 7-3-1 Hongo, Bunkyo-ku, Tokyo 113-8656, Japan \\ ${ }^{4}$ Center for Spintronics Research Network, The University of Tokyo, 7-3-1 Hongo, Bunkyo-ku, Tokyo 113-8656, Japan \\ ${ }^{5}$ Institute of Engineering Innovation, Graduate School of Engineering, The University of Tokyo, 7-3-1 Hongo, \\ Bunkyo-ku, Tokyo 113-8656, Japan \\ ${ }^{6}$ PRESTO, JST, 4-1-8 Honcho, Kawaguchi, Saitama, 332-0012, Japan \\ ${ }^{7}$ New -Technologies: Research Center, University of West Bohemia, Pilsen, Czech Republic \\ ${ }^{8}$ Bacha Khan University, Charsadda, KPK, Pakistan \\ ${ }^{9}$ Deutsches Zentrum für Luft- und Raumfahrt (DLR), Oberpfaffenhofen, 82234 Weßling, Germany \\ ${ }^{10}$ Department of Electrical and Electronic Engineering, Tokyo Institute of Technology, 2-12-1 Ookayama, Meguro-ku, Tokyo 152-0033, Japan \\ ${ }^{11}$ Institute for Solid State Physics, The University of Tokyo, 1-1-1 Koto, Sayo, Hyogo 679-5198, Japan \\ ${ }^{12}$ Department of Physics, The University of Tokyo, 7-3-1 Hongo, Bunkyo-ku, Tokyo 113-0033, Japan \\ ${ }^{13}$ Department of Applied Physics, Waseda University, Okubo, Shinjuku, Tokyo 169-8555, Japan
}

(Received 29 August 2020; revised 14 November 2020; accepted 17 February 2021; published 8 March 2021)

\begin{abstract}
Fully understanding the properties of n-type ferromagnetic semiconductors (FMSs), complementary to the mainstream p-type ones, is a challenging goal in semiconductor spintronics because ferromagnetism in n-type FMSs is theoretically nontrivial. Soft-X-ray angle-resolved photoemission spectroscopy (SX-ARPES) is a powerful approach to examine the mechanism of carrier-induced ferromagnetism in FMSs. Here our SX-ARPES study on the prototypical n-type FMS (In,Fe)As reveals the entire band structure, including the Fe- $3 d$ impurity bands (IBs) and the host InAs ones, and provides direct evidence for electron occupation of the InAs-derived conduction band (CB). A minority-spin Fe-3d IB is found to be located just below the conduction-band minimum (CBM). The IB is formed by the hybridization of the unoccupied Fe $3 d$ states with the occupied CBM of InAs in a spin-dependent way, resulting in the large spin polarization of $\mathrm{CB}$. The band structure with the IB is varied with band filling, which cannot be explained by the rigid-band picture, suggesting a unified picture for realization of carrier-induced ferromagnetism in FMS materials.
\end{abstract}

DOI: 10.1103/PhysRevB.103.115111

\section{INTRODUCTION}

Evolution of information technology has been driven by high-performance electronic devices based on high-quality semiconductor materials. Since the sophistication of semiconductor electronic devices by miniaturization has approached the technical limit, the development of new functional spinbased devices has been pursued [1,2]. Spintronics is a research field aiming at manipulating and utilizing both the charge and spin degrees of freedom of carriers, and spintronic devices have a potential to dramatically reduce the power consumption and realize new functionalities related to the spin degree of freedom [1-5]. For instance, bilayer thin films consisting of a ferromagnetic layer and superconductors, topological insulators, or two-dimensional electron gas have recently attracted much attention for their novel spintronics functionalities, including the realization of Majorana fermions for quan-

*masaki.kobayashi@ee.t.u-tokyo.ac.jp tum computing [3]. To apply these spintronics functionalities to the well-established semiconductor technology, materials having both the semiconducting and ferromagnetic properties are highly desirable. Ferromagnetic semiconductors (FMSs), in which the cation sites in a semiconductor crystal are partially replaced by magnetic atoms, bear a high promise for their applications in semiconductor spintronics [1-3] because of their capability to manipulate both the charge and spin degrees of freedom of carriers [4,5]. FMSs are key materials to realize the practical application of spin-related functionalities [1,4-8].

A fundamental understanding of the origin or mechanism of ferromagnetism in FMSs is important for the application of the FMS materials to spintronics devices. It is known that the ferromagnetism in many FMSs is induced by doping carriers. In contrast to the traditional p-type Mn-doped FMSs such as (Ga,Mn)As, novel Fe-doped III-V FMSs have recently attracted much attention, because Fe-doped FMSs can accommodate both $\mathrm{n}$ - and p-type carriers and exhibit ferromagnetism with high Curie temperature $T_{\mathrm{C}}$ 
(> $300 \mathrm{~K})[5,9,10]$. Using the p-type and/or n-type Fe-doped FMSs, spintronics devices have already been demonstrated $[6,7,11,12] .\left(\operatorname{In}_{1-x}, \mathrm{Fe}_{x}\right)$ As codoped with Be (referred to as (In,Fe)As:Be hereafter) is the first n-type FMS [13,14], where the Be ions act as double donors [15]. (In,Fe)As:Be shows ferromagnetic properties when its electron-carrier concentration $(n)$ is higher than $6 \times 10^{18} \mathrm{~cm}^{-3}$ [13]. The findings of light electron effective mass [14] and large spin-splitting in the conduction-band minimum (CBM) $\Delta_{e x}$ [12] suggest that highly mobile conduction electrons are spin-polarized in the conduction band $(\mathrm{CB})$ of $(\mathrm{In}, \mathrm{Fe}) \mathrm{As}$ :Be. Although the carrier-induced ferromagnetic properties and band structure of (In,Fe)As have been intensively studied so far, the precise mechanism of the ferromagnetism remains an enigma.

Knowledge of the electronic structure, which is composed of the band structure of the host semiconductor and the $3 d$ impurity band, is indispensable for understanding the mechanism of the ferromagnetism in FMSs. Theoretically, it was considered impossible or difficult to realize a zinc-blende n-type FMS due to the weak orbital mixing between the host $s$ and impurity $d$ orbitals [16]. In this article, we have investigated the band structure of the prototypical n-type FMS (In,Fe)As:Be using soft $\mathrm{x}$-ray angle-resolved photoemission spectroscopy (SX-ARPES) and spin-density functional theory (SDFT) calculation to understand the origin of the carrier-induced ferromagnetism. SX-ARPES is one of the most powerful experimental techniques to study the electronic structures of FMSs [17-20]. By using SX-ARPES we reveal the entire band structure of (In,Fe)As:Be, including the $\mathrm{Fe}-3 d$ IB and electron occupation of the InAs-derived CB. Based on the experimental findings, we have identified that the $\mathrm{Fe}-3 d$ IB located near CBM is formed by the hybridization of the CB and the $\mathrm{Fe} 3 d$ state in a spin-dependent way, resulting in the large $s-d$ exchange splitting in (In,Fe)As:Be. The underlying physics of the formation of the IB states in n-type (In,Fe)As is found to be similar to that in p-type (Ga,Mn)As, providing a unified picture of ferromagnetism in FMSs, irrespective of the carrier types. This suggests a materials perspective to realize the carrier-induced ferromagnetism in FMSs.

\section{EXPERIMENTAL}

$\left(\mathrm{In}_{0.95}, \mathrm{Fe}_{0.05}\right) \mathrm{As}: \mathrm{Be},\left(\mathrm{In}_{0.95}, \mathrm{Fe}_{0.05}\right) \mathrm{As}$, and InAs:Be (Bedoped InAs) thin films with a thickness of $20 \mathrm{~nm}$ were grown on InAs(001) substrates at $240^{\circ} \mathrm{C}$ by molecular beam epitaxy. The Be concentrations were $\sim 2 \times 10^{19} \mathrm{~cm}^{-3}$. In order to avoid surface oxidation, the thin films were covered by an amorphous As passivation layer with a thickness of $\sim 1$ $\mathrm{nm}$. An As passivation layer for a GaAs layer hardly induces carrier depletion near the surface of the GaAs layer, and thus band bending is negligible [21]. The SX-ARPES measurements were performed directly through the As capping layer due to the increase of probing depth in the SX region [22]. $T_{\mathrm{C}}$ of the $\left(\mathrm{In}_{0.95}, \mathrm{Fe}_{0.05}\right)$ As:Be film was estimated to be $\sim 40 \mathrm{~K}$ by the Arrott plot of the magnetic circular dichroism measurements. The SX-ARPES experiments were performed at the SX-ARPES endstation [23] of the ADRESS beamline [24]. During the experiments, the samples were cooled to $\sim$ $10 \mathrm{~K}$ by liquid He to suppress the reduction of the coherent $\boldsymbol{k}$-resolved spectral component at high energies. The mea-
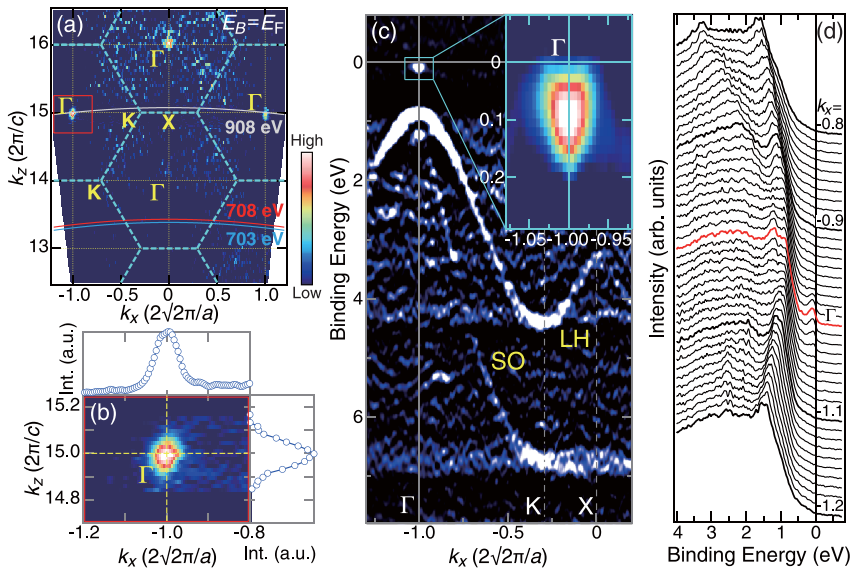

FIG. 1. SX-ARPES spectra of (In,Fe)As:Be. (a) Fermi surface mapping (FMS) in the $k_{z}-k_{x}$ plane. $k_{x}$ indicates the momentum along the $\Gamma-\mathrm{K}-\mathrm{X}$ ([110]) direction. The solid curves are $\boldsymbol{k}$-space cuts for fixed $h v$. (b) Enlarged plot near the $\Gamma$ point $\left[k_{z}=15(2 \pi / c)\right]$. The top and right plots are momentum distribution curves along the $k_{x}$ and $k_{z}$ cuts across the $\Gamma$ point (dashed lines), respectively. (c) ARPES intensity along the $\Gamma-\mathrm{K}-\mathrm{X}$ line obtained at $h v=908 \mathrm{eV}$. The inset is a blowup of the EP. (d) Energy distribution curves (EDCs) around the $\Gamma$ point.

surements were performed mostly at energy resolution $\Delta E \sim$ $120 \mathrm{meV}$. Details of the SDFT calculations are described in Appendix D.

\section{SX-ARPES RESULTS}

\section{A. Fermi surface and band dispersion}

SX-ARPES enables us to reveal bulk band structures of single-crystal materials with sharp definition of the threedimensional electron wave vector $\boldsymbol{k}$ [22]. Figure 1(a) shows the out-of-plane Fermi surface mapping (FSM) in the $k_{x}-k_{z}$ plane of an $\left(\mathrm{In}_{0.95}, \mathrm{Fe}_{0.05}\right)$ As:Be thin film. Here, the $k_{z}$ and $k_{x}$ directions are normal and parallel to the in-plane $\Gamma-\mathrm{K}-\mathrm{X}$ symmetry line of the Brillouin zone, respectively. The mapping shows a tiny but clear Fermi surface (FS) around the $\Gamma$ points. As shown in Fig. 1(a), a clear $k_{z}$ dispersion of all the surface contours excludes the presence of any surface states that are identified by the absence of their $k_{z}$ direction. This ensures that our SX-ARPES spectra truly reflect the bulk electronic structure of $(\mathrm{In}, \mathrm{Fe}) \mathrm{As}: \mathrm{Be}$.

Figure 1(b) shows an enlarged plot of the FS around the $\Gamma$ point $\left[\left(k_{x}, k_{z}\right)=(-1.0,15)(2 \sqrt{2} \pi / a)\right]$. The cross sections of the FS in the $k_{x}-k_{z}$ out of plane as well as that in the $k_{x}-k_{y}$ in plane (not shown) are circular. Actually, the Fermi momenta are about 0.12 and $0.13 \AA^{-1}$ for the $k_{x}$ and $k_{z}$ directions, respectively. This suggests that the shape of the FS is spherical within experimental accuracy. The carrier concentration $n$ estimated from the volume of the FS is $\sim 2 \times 10^{19} \mathrm{~cm}^{-3}$; this value is above the threshold $\left(6 \times 10^{18} \mathrm{~cm}^{-3}\right)$ to induce the ferromagnetism in (In,Fe)As [13], and it is in good agreement with $n$ estimated from the Hall measurement and is of the same order of magnitude as the concentration of codoped $\mathrm{Be}$. This result is consistent with the assumption that the $\mathrm{Be}$ dopant acts as a double donor in $(\mathrm{In}, \mathrm{Fe}) \mathrm{As}$. 

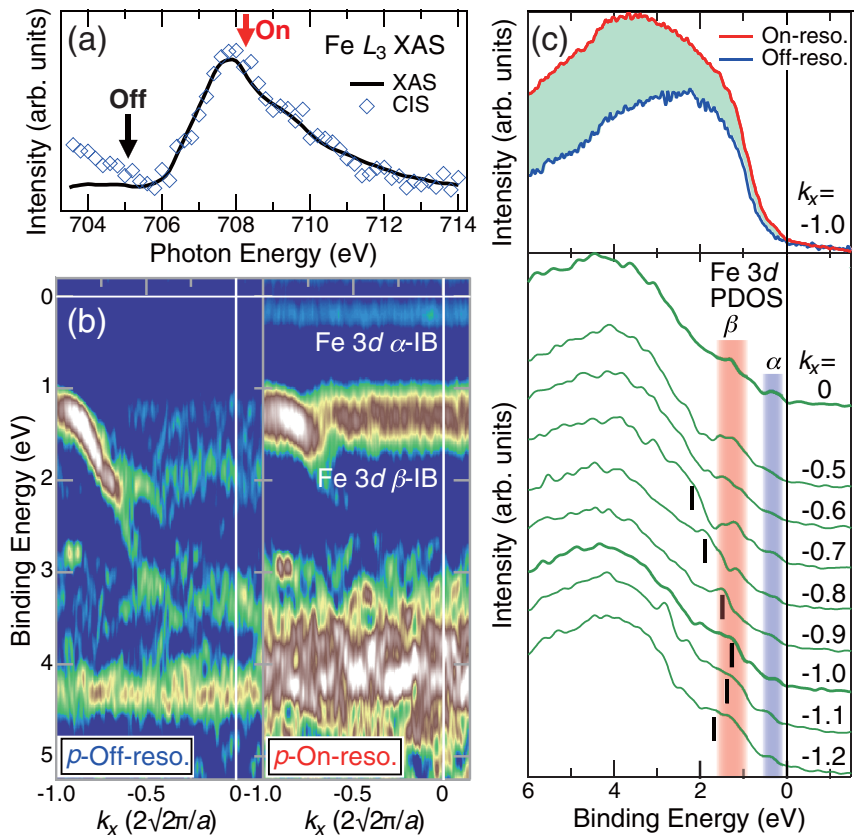

FIG. 2. Resonant ARPES spectra of (In,Fe)As:Be. (a) Fe $L_{3}$ XAS spectrum. The constant-initial-state (CIS) spectrum at $E_{B}=0.25 \mathrm{eV}$ corresponding to the $\alpha$-IB is also plotted. The arrows denote the excitation energies for rARPES. (b) Fe $L_{3}$ rARPES spectra. The left and right panels are the off- and on-resonance spectra, respectively. $\alpha$ - and $\beta$-IBs are Fe $3 d$-derived impurity bands. (c) EDCs of the rARPES spectra. (Top) On- and off-resonance EDCs at $k_{x}=-1.0$. (Bottom) Differences of the EDCs corresponding to the Fe-3d PDOS at various $k_{x}$. The vertical bars indicate a dispersive peak.

Figure 1(c) shows the band dispersion along the $\Gamma-\mathrm{K}-\mathrm{X}$ line. Here the incident photon energy $h v$ is set at $908 \mathrm{eV}$ in order to bring the $k_{z}$ value to the $\Gamma$ point [see Fig. 1(a)]. The holelike band dispersions centered at $\Gamma$ are the light-hole (LH) and split-off (SO) bands [see Appendix A for the heavy-hole $(\mathrm{HH})$ band], and the electronlike one near the Fermi level $\left(E_{\mathrm{F}}\right)$ identifies the CB. As shown in Figs. 1(c) and 1(d), the CB dispersion clearly crosses $E_{\mathrm{F}}$ and forms the small FS so-called electron pocket (EP), consistent with the n-type conductivity confirmed by Hall-effect and thermoelectric Seebeck-effect measurements [13]. These results demonstrate that the electronic transport in ferromagnetic $(\mathrm{In}, \mathrm{Fe}) \mathrm{As}$ :Be arises from the electron carriers in the CB originating from the $s$ band of InAs. This naturally explains their light effective mass [14] and the long mean free path of electron carriers [11].

\section{B. Fe 3d-derived impurity band in the band structure}

In addition to the band dispersions, the location of the $\mathrm{Fe}-3 d$ IB is also important to understand the ferromagnetism in $(\mathrm{In}, \mathrm{Fe}) \mathrm{As}$. To identify the $\mathrm{Fe}-3 d \mathrm{IB}$, we have employed resonant ARPES (rARPES) at the Fe $L_{3}$ edge. The resonant enhancement of the photoemission intensity occurs at $h v$ corresponding to the peaks of the Fe $L_{3}$ X-ray absorption spectroscopy (XAS) spectrum [Fig. 2(a)]. Figure 2(b) shows the on- and off-resonance ARPES spectra measured with $h v=708.2 \mathrm{eV}$ [the red arrow in Fig. 2(a)] and $705 \mathrm{eV}$ (the black arrow), respectively. Note that in addition to the intense Fe-3 3 IB ( $\beta$-IB), a weak Fe-3 3 IB ( $\alpha$-IB) appears near the CBM. The intensity of the $\alpha$-IB is resonantly enhanced when $h v$ is at the peak of the XAS spectrum [see Fig. 2(a)], indicating that the $\alpha$-IB is derived from the Fe $3 d$ orbitals in ( $\mathrm{In}, \mathrm{Fe}) \mathrm{As}$, not from extrinsic Fe oxides. The $\beta$-IB results from the hybridization of the Fe $3 d$ orbitals with the valence band (VB) and appears in the vicinity of the valence-band maximum (VBM). While the Mn-3d IB in (Ga,Mn)As splits off from VBM due to the strong $p$ - $d$ hybridization [17], the $\mathrm{Fe}-3 d \beta$-IB in (In,Fe)As does not. To elucidate the hybridization between the $\mathrm{Fe} 3 d$ orbital and the ligand experimentally, the Fe-3 $d$ partial density of states (PDOS) corresponding to the difference between the on- and off-resonance spectra are plotted in Fig. 2(c). In addition to the $\boldsymbol{k}$-independent $\alpha$-IB and $\beta$-IB, the PDOS around $k_{x}=-1.0(2 \sqrt{2} \pi / a)$ shows a dispersive feature corresponding to the LH band (marked by vertical bars). Since the Fe $3 d$ orbitals are independent of $\boldsymbol{k}$, the observation of dispersion in the PDOS evidences that the $\mathrm{LH}$ band is also resonantly enhanced at the Fe $L_{3}$ absorption edge through hybridization with the $\mathrm{Fe} 3 d$ orbital.

\section{Band structures of (In,Fe)As with and without carrier doping}

The band structure of ( $\mathrm{In}, \mathrm{Fe}) \mathrm{As}: \mathrm{Be}$ revealed by $\mathrm{SX}$ ARPES is summarized in Fig. 3(a), where the band dispersions along the $\Gamma-\mathrm{K}-\mathrm{X}$ line are superimposed with the $\mathrm{Fe}-3 d$ IBs. To separate the effects of Fe doping on the band structure of (In,Fe)As from that of Be, we have measured SX-ARPES spectra of the parent compound InAs:Be, as presented in Fig. 3(b). The EP around $\Gamma$ also exists in InAs:Be. Comparing the band dispersions between InAs:Be and (In,Fe)As:Be, we find them nearly identical except for the presence of IBs in (In,Fe)As:Be. These results suggest that the electron carriers in $(\mathrm{In}, \mathrm{Fe}) \mathrm{As}$ :Be originate predominantly from the Be codoping and not from the $\mathrm{Fe}$ doping. This indicates that the $\mathrm{Fe}^{3+}$ ions substitute for the In cation sites and provide the system with only the magnetic moments. To reveal the $n$ dependence of the band structure, a paramagnetic $\left(\operatorname{In}_{0.95}, \mathrm{Fe}_{0.05}\right)$ As film without Be doping has been measured. Figure 3(c) shows the band structure measured on (In,Fe)As. One can see that both the EP and the $\alpha$-IB disappear in the (In,Fe)As sample with a lower $n$. If the rigid-band model is applicable, because the position of $E_{\mathrm{F}}$ is at or slightly below CBM in (In,Fe)As, the $\alpha$-IB located just below CBM would be occupied, but it is not the case [see Fig. 3(c)]. The present observation suggests that the location of the $\alpha$-IB depends on $n$.

Based on these experimental findings, schematic views of the density of states (DOS) of ferromagnetic (In,Fe)As:Be and paramagnetic (In,Fe)As are shown in Figs. 4(a) and 4(b), respectively. It should be mentioned here that the concentration of the Be dopant is one to two orders of magnitude smaller than that of the Fe ions. If an in-gap Fe-3 $d$ IB exists below CBM, the amount of the supplied electrons by the $\mathrm{Be}$ doping was insufficient to fully occupy the IB and $E_{\mathrm{F}}$ would be located in the IB, not in the CB. Considering the appearance of the $\alpha$-IB depending on $n$, the electron density of the $\alpha$-IB located below $E_{\mathrm{F}}$ is presumably the same order of the $\mathrm{Be}$ dopant $\left(10^{19} \mathrm{~cm}^{-3}\right)$, suggesting the partial occupation of the $\alpha$ IB as shown in Fig. 4(a). This phenomenon looks analogous to the evolution of an IB state located below $E_{\mathrm{F}}$ with increasing 


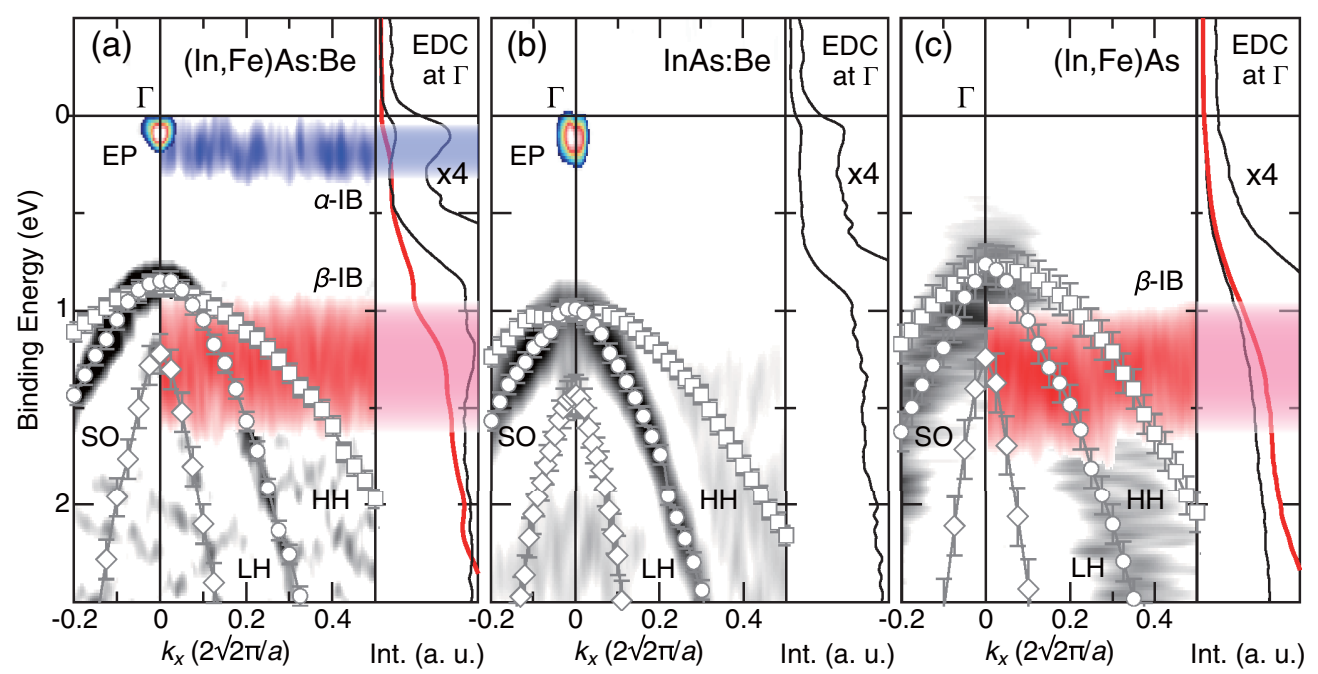

FIG. 3. Comparison of band structures of (In,Fe)As with and without doping. (a) Band dispersions along the $\Gamma-\mathrm{K}-\mathrm{X}$ ([110]) line in (In,Fe)As:Be. The IB intensity obtained by rARPES is superimposed in the positive $k_{x}$ region. The dispersions of the LH (circle), HH (square), and SO (rhombus) bands are also plotted. (Right) EDC at $\Gamma$ (thin line) and Fe-3d PDOS (thick line). The colored areas denote the energy ranges of the Fe-3d IBs. (b) Band dispersion near $E_{\mathrm{F}}$ in InAs:Be, with the EDC at $\Gamma$ on the right. (c) Band dispersion in (In,Fe)As as well as (In,Fe)As:Be (Fig. 3(a)).

$\mathrm{K}^{+}$concentration in $\mathrm{K}_{x} \mathrm{Co}_{60}[25,26]$. A fundamental question here is why the partially filled $\alpha$-IB is located below CBM in $(\mathrm{In}, \mathrm{Fe}) \mathrm{As}: \mathrm{Be}$ despite the electron-carrier occupation of CBM.

\section{SDFT calculations for (In,Fe)As}

As shown in Fig. 2(a), the Fe $L_{3}$ XAS is characterized by a smooth and broad spectral peak without multiplet structure, which is similar to that of Fe metal or metallic Fe compounds (see Appendix B for details). Because the Fe $3 d$ states in (In,Fe)As:Be are likely to be strongly covalent with ligand As $p$ orbitals and are different from purely ionic $\mathrm{Fe}^{3+}$ [27], the broad spectral feature reflects the strong hybridization of the $\mathrm{Fe} 3 d$ states with the ligand. As shown in Fig. 7 (see Appendix C), the SDFT calculations have demonstrated that (a)

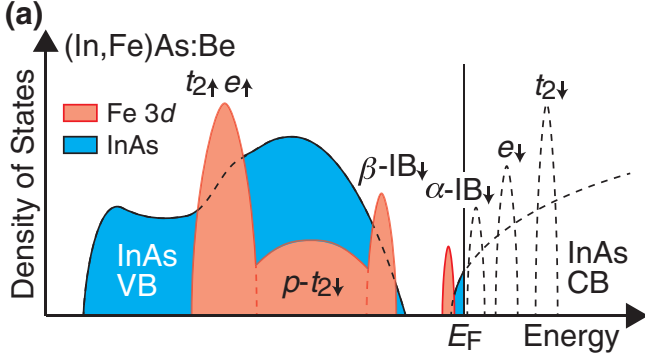

(c)

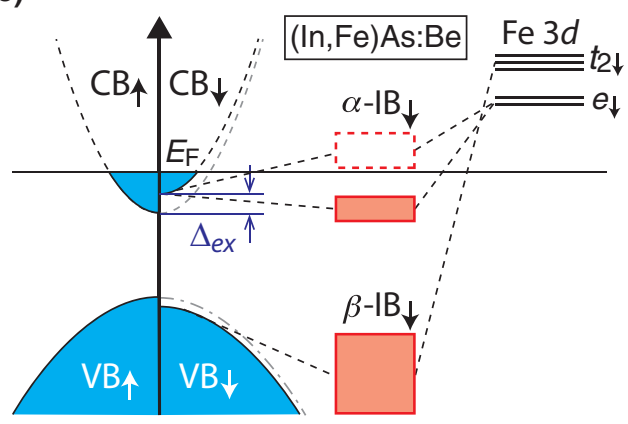

(b)

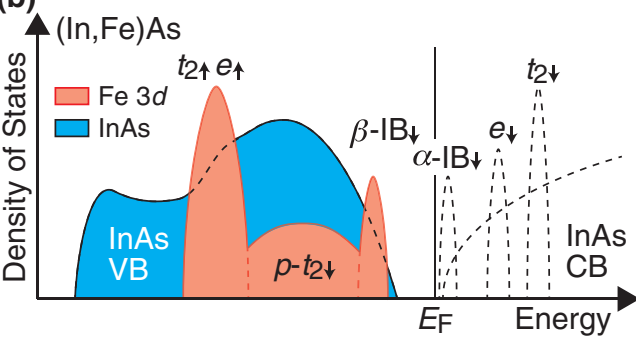

(d)

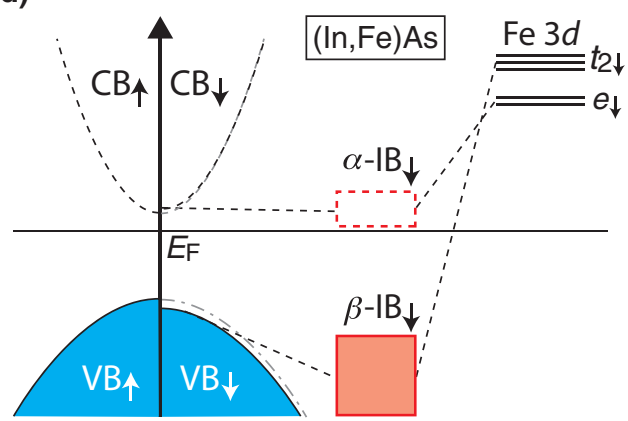

FIG. 4. Band diagram of (In,Fe)As. (a, b) Schematic DOS for ferromagnetic (In,Fe)As:Be and paramagnetic (In,Fe)As, respectively. DOS including the Fe $3 d$-derived IBs embedded in the VB and CB of the host InAs. The expected unoccupied Fe $3 d_{\downarrow}$ states are also shown. (c, d) Band diagrams for (In,Fe)As:Be and (In,Fe)As, respectively. Hybridization between Fe $3 d e_{\downarrow}$ and CB leads to the formation of $\alpha$-IB and hybridization between Fe $3 d t_{2 \downarrow}$ and VB to the formation of $\beta$-IB. Dash-dotted lines are the InAs-derived band dispersion without the hybridization. Electron occupation of CB pushes down the partially filled $\alpha$-IB to be located below CBM, leading to the large spin-splitting $\Delta_{e x}$ of the CB. 
the Fe $3 d$ minority-spin $(\downarrow)$ states strongly hybridize with the ligand VB and CB states. Only the minority-spin states of the ligands hybridize with the unoccupied Fe $3 d$ states, because the majority-spin ( $\uparrow) 3 d$ states are fully occupied and located well below the host VB. The $\beta$-IB originates from the hybridization between the Fe $3 d_{\downarrow}$ states and the VBM states. Note that the calculated $e_{\downarrow}$ states show a slightly dispersive feature, indicating finite hybridization of the $e_{\downarrow}$ states with the minority-spin host band states. This result indicates that the hybridization between the $e_{\downarrow}$ and CBM states forms the $\alpha$-IB just below CBM as shown in Fig. 4(d), if the hybridization is strong enough.

\section{DISCUSSION}

\section{A. Formation of the Fe $3 d$ IB through the $s-d$ hybridization}

When the CB is partially occupied, it is probable that the $\alpha$-IB splits off from CBM through the $s-e_{\downarrow}$ hybridization, leading to the energy gap between the occupied and unoccupied $\alpha$-IB states, as shown in Fig. 4(c). This partially filled split-off IB below $E_{\mathrm{F}}$ is similar to the formation of IB in the band gap observed in $\mathrm{K}_{x} \mathrm{C}_{60}$, where the $\mathrm{IB}$ is derived from electrons donated into the lowest unoccupied molecular orbital by the potassium [25,26]. Additionally, emergence of the split-off IB related to the carrier-induced ferromagnetism is a common feature to p-type FMS (Ga,Mn)As [17]. It should note here that the $s-e_{\downarrow}$ hybridization is spin-dependent in (In,Fe)As, that is, it happens only for the minority-spin states because the majority-spin states of $\mathrm{Fe} 3 d$ are fully occupied. With the partially filled split-off $\alpha$-IB, the minority-spin CBM loses states whereas the majority-spin one does not, as shown in Fig. 4(c).

The formation of the split-off state in the spin-dependent way results in a large spin-splitting $\Delta_{e x}$ of CBM in (In,Fe)As, as shown in Fig. 4(c). Indeed, the spin-splitting $\Delta_{e x}$ as large as 30-50 meV has been observed in n-(In,Fe)As/p-InAs Esakidiode structures [12]. Based on the experimental findings, we have discussed qualitatively the origin of the large $s-d$ exchange interaction. According to the Anderson Hamiltonian [28], the $s$ - $d$ exchange interaction $N_{0} \alpha$ is given by $\left|N_{0} \alpha\right|=-2\left|V_{s d}\right|^{2}\left(\frac{1}{E_{C}-\varepsilon_{d}}+\frac{1}{U-E_{C}+\varepsilon_{d}}\right)$, where $E_{C}$ is the energy of CBM, $\varepsilon_{d}$ is the energy of the $d$ states, $U$ is the Coulomb repulsion between the $d$ electrons, and $V_{s d}$ is the $s-d$ mixing potential [29]. From the value of $T_{\mathrm{C}},\left|N_{0} \alpha\right|$ for (In,Fe)As has been estimated to be $\sim 2.8 \mathrm{eV}$ [14], which is much larger than the $p$ - $d$ exchange interaction $\left|N_{0} \beta\right| \sim 1.2 \mathrm{eV}$ in (Ga,Mn)As [30]. Since $\left|N_{0} \alpha\right|$ is inversely proportional to the energy difference $\left|E_{C}-\varepsilon_{d}\right|$ or $\left|U-E_{C}+\varepsilon_{d}\right|$, if one of the energy differences approaches zero, the value of $\left|N_{0} \alpha\right|$ will be significantly enhanced. This condition is referred to as the "resonance" condition [14]. As discussed above, while $\varepsilon_{d}$ is well above $E_{C}$, the energy difference $\left|U-E_{C}+\varepsilon_{d}\right|$ is possibly small to satisfy the resonance condition in (In,Fe)As:Be. It follows from the above argument that the strong $s$ - $d$ exchange interaction $\left|N_{0} \alpha\right|[12,14]$, leading to the carrierinduced ferromagnetism in (In,Fe)As:Be, originates from the hybridization between the $e_{\downarrow}$ and CBM states, consistent with the large $\Delta_{e x}$ observed by tunneling spectroscopy [12].

\section{B. Materials perspective for ferromagnetic semiconductors}

The present results put forward important aspects for the materials design of FMSs. Generally, the ionicity of doped transition-metal ions will increase with the band gap of the host semiconductor [31]. Considering this tendency, the spindependent hybridization with the ligand would hardly occur in an FMS with a wide-gap semiconductor. On the other hand, it has been pointed out theoretically that narrow-gap III-V semiconductors with strong covalency are appropriate for host compounds of both p-type and n-type FMSs [32,33]. This scenario is consistent with the observations that n-type FMS (In,Fe)Sb with a smaller band gap shows higher $T_{\mathrm{C}}$ than (In,Fe)As:Be [10], and p-type $(\mathrm{Ba}, \mathrm{K})(\mathrm{Zn}, \mathrm{Mn})_{2} \mathrm{As}_{2}$ has higher $T_{\mathrm{C}}$ than (Ga,Mn)As [34]. Since the split-off IB states originate from strong $s$ - $d$ or $p$ - $d$ hybridization, the band structures proposed in the present study are likely to be applicable to other FMSs based on narrow-gap semiconductors. Another important aspect here is that the band structure with the split-off IB changes with $n$. Then, additional carrier doping is necessary to validate realization of carrier-induced ferromagnetism in FMSs aside from the transition-metal dopants acting as donors or acceptors like $\mathrm{Mn}$ ions in (Ga,Mn)As. For example, Mn-doped FMSs with narrow-gap II-VI semiconductors codoped with donor dopants will be candidates for n-type FMSs. Many FMS materials without carrier doping have been found to be paramagnetic so far. The emergence of carrier-induced ferromagnetism in such FMSs should be reexamined with additional carrier doping.

One of the most intriguing features here is that the energy position of the $\alpha$-IB depends on the CB filling, which means that the rigid-band picture is not applicable. The split-off $\alpha$-IB through the $s$ - $d$ hybridization in n-type (In,Fe)As discussed below is analogous to the split-off IB through the $p$ - $d$ hybridization in p-type (Ga,Mn)As [17], although the carrier type is different. Note that the strong hybridization is essential to form the split-off IB states in both the FMSs. As to the underlying physics, the formation of these IB states due to hybridization with ligands is also analogous to that of the Zhang-Rice singlet band [35,36]. It is likely that the split-off IB can be formed in a spin-dependent way depending on $n$ and strength of the $s p$ - $d$ hybridization. These arguments suggest that appearance of the hybridization-derived split-off IB states near the band edge of host semiconductor (VBM for p-type or CBM for n-type) is attributed to be a unified picture to realize carrier-induced ferromagnetism in FMS materials irrespective of the carrier type. To obtain further evidence for the unified picture for the carrier-induced ferromagnetism, systematic studies of p-type and n-type FMSs are desirable.

\section{CONCLUSION}

In conclusion, we have conducted SX-ARPES measurements combined with SDFT calculations for the prototypical n-type FMS (In,Fe)As:Be in order to elucidate the nature of the n-type FMSs. The SX-ARPES reveals the entire band structure, including the Fe-3 $d$ IBs and the host InAs bands, providing direct evidence for electron occupation of the InAsderived CB. The Fe-3d IB located near CBM is formed through the hybridization with the ligand, which is the origin 
of the carrier-induced ferromagnetism in n-type (In,Fe)As. When the electron carriers occupy the $\mathrm{CB}$, the partially filled IB splits off from CBM, leading to the large spin polarization of the CB. The intriguing feature that its band structure is deformed depending on the band filling is unexpected from the ordinary rigid-band picture. The underlying physics of the formation of the split-off IB is found to be similar to that in p-type (Ga,Mn)As [17], suggesting a unified picture for realizing carrier-induced ferromagnetism in FMSs irrespective of the carrier types.

\section{ACKNOWLEDGMENTS}

This work was supported by Grants-in-Aid for Scientific Research (Grants No. 15H02109, No. 17H04922, No. 18H05345, and No. 20H05650) from JSPS, and the CREST (Grant No. JPMJCR1777) and PRESTO Programs (Grant No. JPMJPR19LB) of the Japan Science and Technology Agency. J.M. and W.K. would like to thank the CEDAMNF (Project No. CZ.02.1.01/0.0/0.0/15_003/0000358), cofunded by the Ministry of Education, Youth, and Sports of the Czech Republic. This work was partially supported the Spintronics Research Network of Japan (Spin-RNJ). M.K. acknowledges support from the Japan Society for the Promotion of Science.

\section{APPENDIX A: LINEAR POLARIZATION DEPENDENCE OF THE ARPES SPECTRA}

The intensity of band dispersion depends on the polarization of the incident $x$ rays reflecting the matrix element effects [37] and symmetry of the bands: The LH and SO bands, and CBM of the host InAs can be observed with $p$ polarization, and the heavy-hole $(\mathrm{HH})$ band with $s$ polarization, as well as the polarization dependence of bands of GaAs [23,38] (note, however, that the CBM of GaAs has not been observed in the previous study because $E_{\mathrm{F}}$ is located below the CBM in p-type GaAs). Figure 5 shows the ARPES spectrum along the $\Gamma-\mathrm{K}-\mathrm{X}$ symmetry line taken with the $s$ polarization. Only the $\mathrm{HH}$ band is clearly observed due to the matrix element effects. Since the linear polarization dependence of ARPES reflects the symmetry of the bands [37], the CBM observed only with $p$ polarization has the same symmetry as the LH and $\mathrm{SO}$ bands, not as the $\mathrm{HH}$ band. Figure 6 shows $\mathrm{Fe} L_{3}$ resonant ARPES spectra taken with the $s$ polarization. Both the Fe $3 d$ $\alpha$ - and $\beta$-IBs can be observed in the on-resonance spectrum, while the intensities of the IBs are small and only the HH band can be observed in the off-resonance spectrum. Both the $\alpha$-IB and $\beta$-IB are active for the $p$ and $s$ polarizations, as shown in Figs. 2(b) and 6, indicating that the IBs possibly hybridize with the LH, HH, and SO bands of InAs.

\section{APPENDIX B: SPECTRAL LINE SHAPE OF Fe $L_{3}$ XAS}

As shown in Fig. 2(e), the Fe $L_{3}$ XAS spectrum shows a single peak at around $708 \mathrm{eV}$ with a small dip around $h v \sim$ $709.5 \mathrm{eV}$, different from multiple structures observed in $\mathrm{Fe}$ oxides [39], and the spectral line shape resembles those of Fe metal [40] and Fe pnictides [41]. The preedge structure at around $h v \sim 705 \mathrm{eV}$ comes from the In $M_{2}$ edge. Although the spectral feature is similar to that of Fe metal, our previous
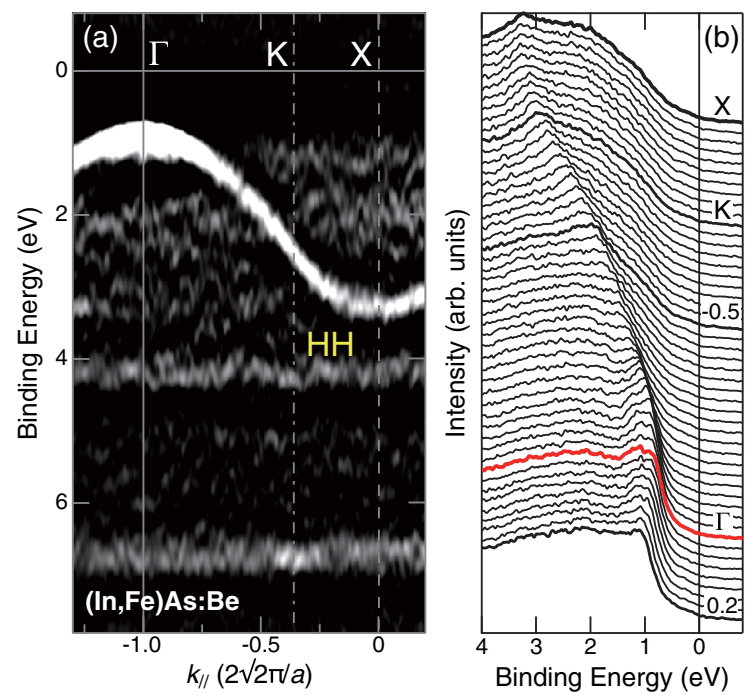

FIG. 5. APRES spectrum of (In,Fe)As:Be obtained along the $\Gamma$ $\mathrm{K}-\mathrm{X}$ line with $s$ polarization. (a) APRES intensity along the $\Gamma-\mathrm{K}-\mathrm{X}$ line obtained at $h v=908 \mathrm{eV}$. HH is the heavy-hole band of the host InAs. The incident photon energy for this measurement is of $908 \mathrm{eV}$ as well as the ARPES spectrum taken with $p$ polarization shown in Fig. 2(c). (b) EDCs around the $\Gamma$ point.

X-ray magnetic circular dichroism (XMCD) study confirms that the electronic structure of $\mathrm{Fe}$ in (In,Fe)As is different from Fe metal [27], excluding the possibility that the ferromagnetic property comes from aggregation of $\mathrm{Fe}$ metal clusters in (In,Fe)As. The negligibly weak signal of Fe oxide (at $h v \sim$ $709.5 \mathrm{eV}$ ) indicates that the amorphous As passivation layer well protected the $(\mathrm{In}, \mathrm{Fe}) \mathrm{As}$ :Be layer from oxidation.

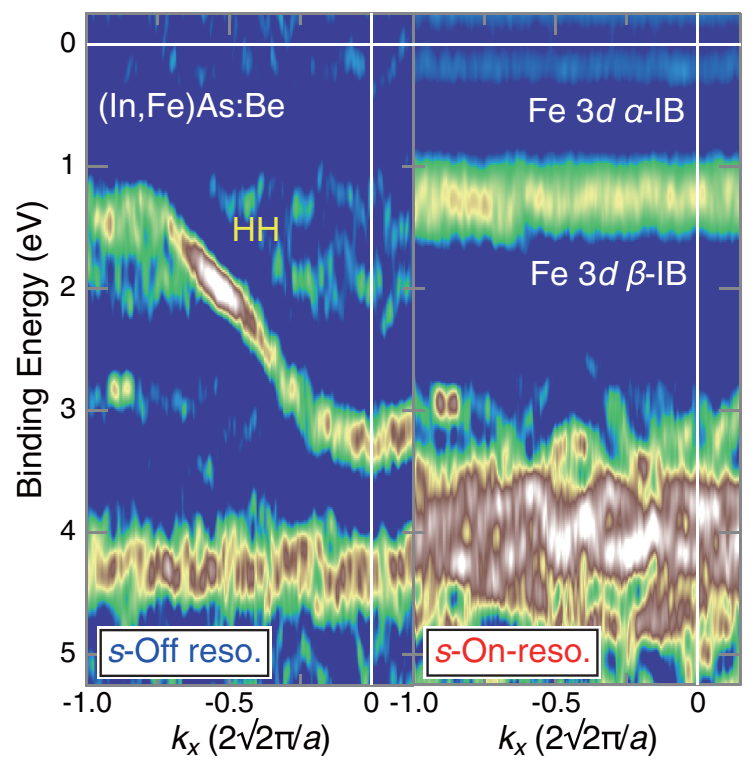

FIG. 6. Fe $L_{3}$ resonant ARPES spectra of (In,Fe)As:Be taken with $s$ polarization. The left and right panels are the off- and onresonance spectra, respectively. IB is the $\mathrm{Fe} 3 d$-derived impurity band. 

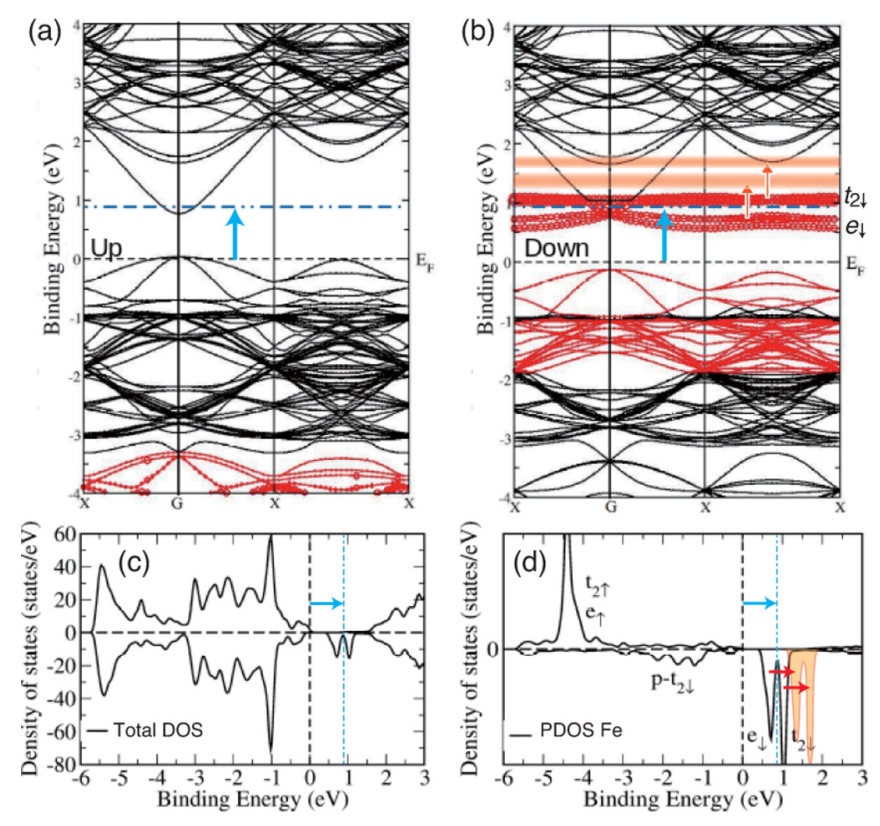

FIG. 7. Spin-density functional theory calculation for an $\mathrm{In}_{31} \mathrm{Fe}_{1} \mathrm{As}_{32}$ supercell. (a, b) Spin-resolved band structure. The weight of $\mathrm{Fe} 3 d$ is indicated by the size of red circles. The blue dash-dotted line is the energy position of $E_{\mathrm{F}}$ in the Be-doped samples as observed experimentally. Note that as $E_{\mathrm{F}}$ is shifted upwards, the Fe $3 d t_{2 \downarrow}$ and $e_{\downarrow}$ bands are also shifted upwards and remain located above $E_{\mathrm{F}}$ due to Coulomb interaction, as denoted by red arrows. The impurity band near $E_{\mathrm{F}}(\alpha$-IB) observed in the present work is considered to result from hybridization between the Fe $e_{\downarrow}$ bands and the CBM. (c) DOS for the up-spin (upper curve) and down-spin states (lower curve). (d) Partial density of states for $\mathrm{Fe} 3 d$.

\section{APPENDIX C: SPIN-DENSITY FUNCTIONAL THEORY CALCULATIONS}

To gain a basic insight into the band structure of (In,Fe)As:Be, we have performed SDFT calculations for the band structures of (In,Fe)As. Figures 7(a) and 7(b) show the calculated band dispersions of (In,Fe)As for spin-up (majority) and spin-down (minority) states, respectively. Here we focus on the spin-polarized states and their hybridization revealed by the SDFT calculation. The Fe $3 d e$ minority-spin $\left(e_{\downarrow}\right)$ band appears near the CBM in the calculation. Note that this $e_{\downarrow}$ band shows a weak but finite dispersion, suggesting that the CBM states hybridize with the Fe $3 d$ orbitals. Comparison with experiment as discussed below indicates that the observed $E_{\mathrm{F}}$ is located just above the CBM (dash-dotted lines). Concomitantly, the down-spin states $\left(e_{\downarrow}\right.$ and $\left.t_{2 \downarrow}\right)$ should be shifted upwards to above $E_{\mathrm{F}}$ due to the on-site Coulomb interaction at the $\mathrm{Fe}$ site, which is necessary to keep the Fe $d$-electron count within a realistic range but cannot be properly taken into account in SDFT. Also, an IB originating from the hybridization between the $e_{\downarrow}$ and CBM states appears below $E_{\mathrm{F}}$. In this scenario, the spin direction of the $\mathrm{Fe}-3 d$ IB is opposite to the total magnetization, consistent with the observation of the spin-Esaki-diode behavior using (In,Fe)As [12]. As shown in Fig. 1(b), the Fe $3 d t_{2}$ minorityspin states strongly hybridize with the VB states of the InAs host below $E=-2 \mathrm{eV}$ (the $p-t_{2 \downarrow}$ state). To see quantitatively how strongly the Fe $3 d$ states are hybridized with the InAs
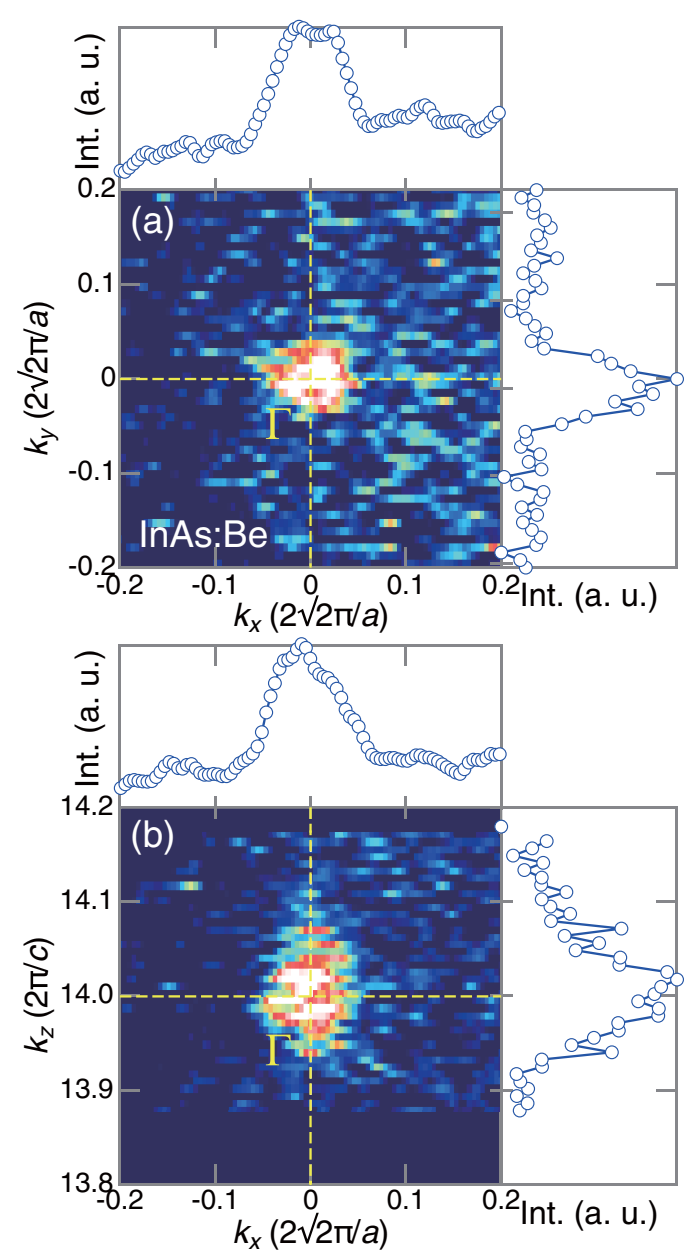

FIG. 8. Fermi surface mapping of an InAs:Be thin film. (a, b) In-plane and out-of-plane FSM, respectively. The top right plots are momentum distribution curves (MDCs) along the $k_{x}$ and $k_{y}$ or $k_{z}$ cuts across the $\Gamma$ point (dashed lines), respectively.

band, the total DOS and Fe $3 d$ PDOS are plotted in Figs. 7(c) and $7(\mathrm{~d})$, respectively. The Fe $3 d$ minority-spin states obviously contribute to the VBM states, while the majority-spin band is largely located in the lower part of the VB (well below $E=-3.5 \mathrm{eV}$ ) and has only negligible contributions to the VBM. Therefore the SDFT calculation indicates that the minority-spin Fe $3 d$ states strongly hybridize with the ligand VB and conduction band. As discussed in the main text, the result that the dispersive feature of the $e_{\downarrow}$ band originating the hybridization with the $\mathrm{CB}$ is a key to understand the band structure of (In,Fe)As:Be and the appearance of the $\alpha$-IB.

\section{APPENDIX D: DETAILS OF THE CALCULATION}

For the SDFT calculation of the electronic structure of $\mathrm{In}_{0.9} \mathrm{Fe}_{0.1} \mathrm{As}$, we applied the all-electron augmented plane wave + local orbitals WIEN2K code. We employ the EngelVosko parameterization of a generalized gradient approximation (EV-GGA) [42]. This function has been successfully used in the past in studies of electronic band structure and density of states of InAs. Calculations for $\operatorname{In}_{1-x} \mathrm{Fe}_{x} \mathrm{As}$ has been performed within the supercell of 64 atoms, which nominally corresponds to $x=0.3125$. The Brillouin zone 

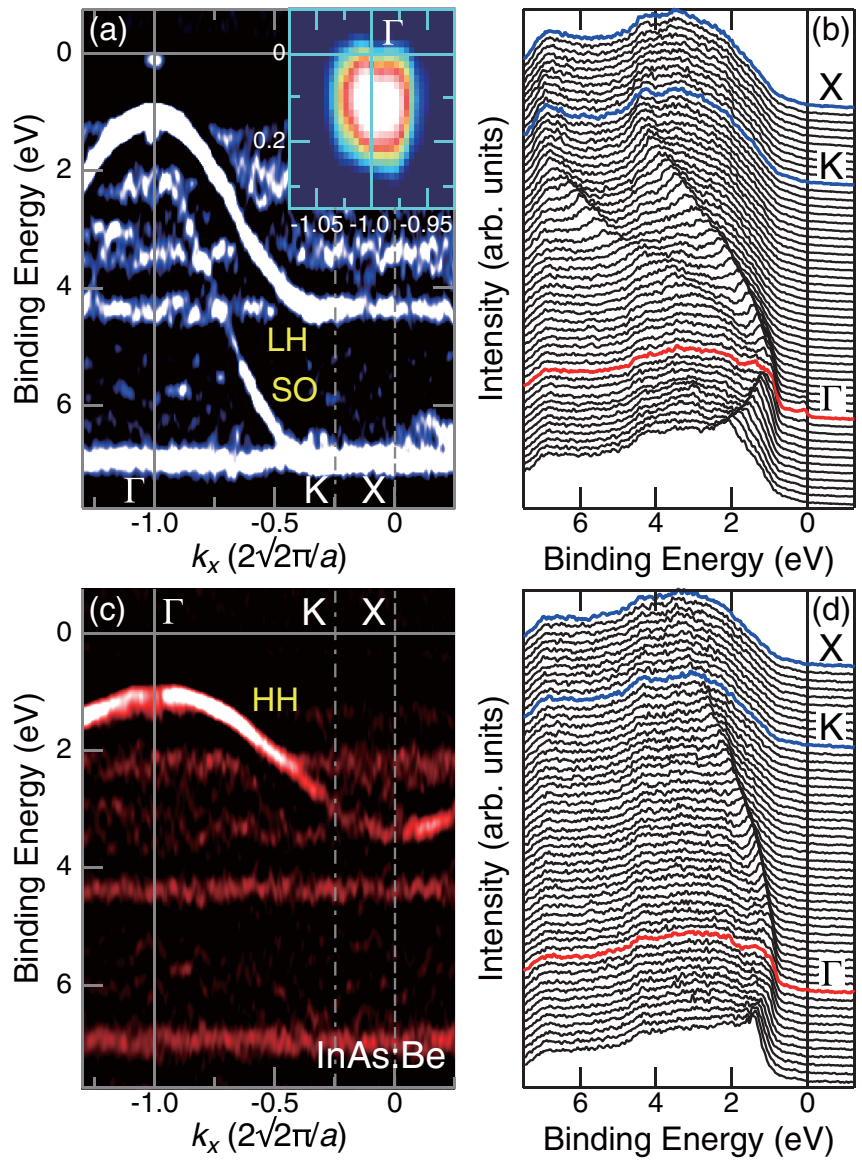

FIG. 9. Band dispersions of InAs:Be. (a) ARPES image plot along the $\Gamma-\mathrm{K}-\mathrm{X}$ line obtained with $p$ polarization. $\mathrm{LH}$ and SO denote the light-hole and split-off bands, respectively. (b) Energy distribution curves (EDCs) of the spectrum taken with $p$ polarization. (c) ARPES image plot along the $\Gamma-\mathrm{K}-\mathrm{X}$ line obtained with $s$ polarization. HH denotes the heavy-hole band. (d) EDCs of the spectrum taken with $s$ polarization. The ARPES images are second derivatives of the ARPES intensities.

was sampled by $5 \times 5 \times 5$ mesh. The wave functions in the atomic spheres were expanded up to an angular momentum of $l_{\max }=10$. The plane-wave cutoff in the interstitial region was set so that $R_{\mathrm{MT}} \times K_{\mathrm{MAX}}=7$, and the energy and charge convergence was set to $10^{-4} \mathrm{eV}$. Here $R_{\mathrm{MT}}$ and $K_{\mathrm{MAX}}$ are the smallest atomic sphere radius and the largest $K$ vector of the plane-wave expansion of the wave function, and the product $R_{\mathrm{MT}} \times K_{\mathrm{MAX}}$ describes the quality of the basis set used in the linearized augmented plane-wave (LAPW) method.

\section{APPENDIX E: SX-ARPES SPECTRA OF InAs:Be}

The SX-ARPES spectra of an InAs:Be thin film was measured to compare with those of the ferromagnetic (In,Fe)As:Be thin film. Figure 8 shows the Fermi surface mapping of the InAs:Be film. The in-plane and out-of-plane FSMs indicate that the shape of the electron Fermi surface in InAs:Be is spherical within experimental accuracy, as for (In,Fe)As:Be shown in Fig. 1(b). Its Fermi momentum in the InAs:Be film is 1.16 times larger than that in the $(\mathrm{In}, \mathrm{Fe}) \mathrm{As}$ :Be film, indicating that the electron-carrier concen-
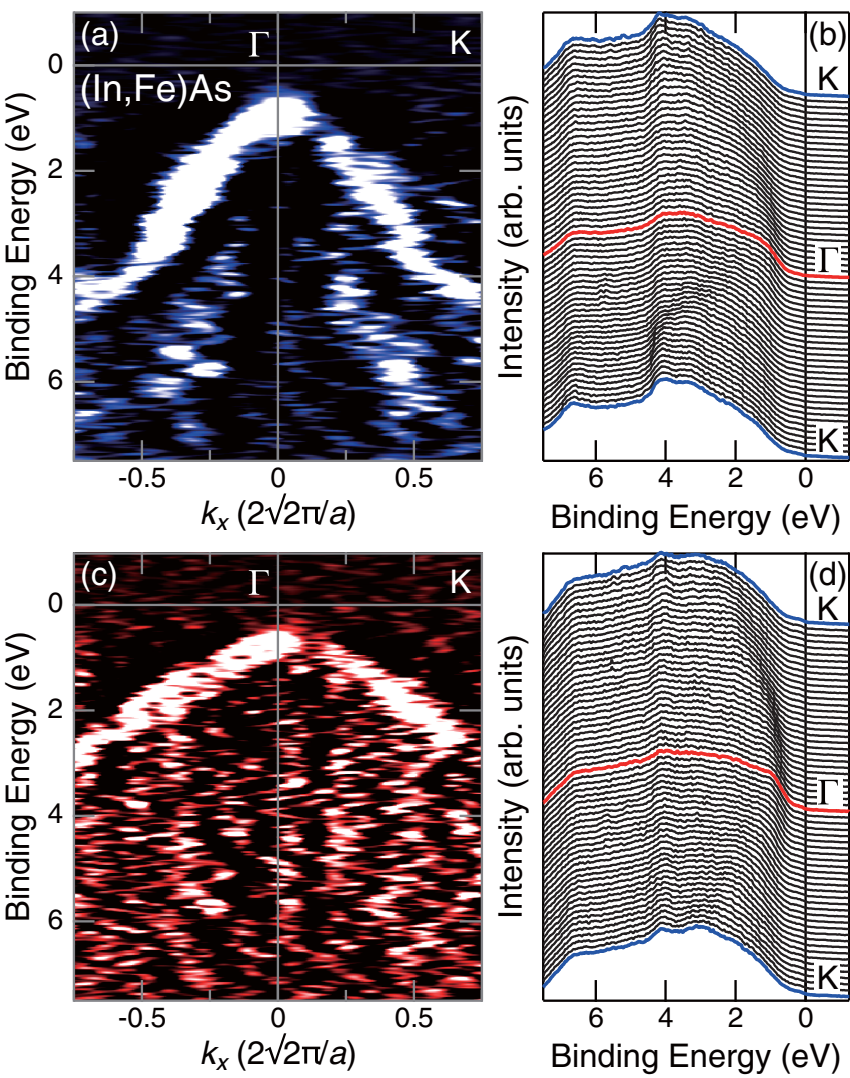

FIG. 10. Band dispersions of paramagnetic (In,Fe)As. (a) ARPES image plot along the $\Gamma-\mathrm{K}-\mathrm{X}$ line obtained with $p$ polarization. (b) EDCs of the spectrum taken with $p$ polarization. (c) ARPES image plot along the $\Gamma-\mathrm{K}-\mathrm{X}$ line obtained with $s$ polarization. $\mathrm{HH}$ denotes the heavy-hole band. (d) EDCs of the spectrum taken with $s$ polarization. The ARPES images are second derivatives of the ARPES intensities.

tration is $\sim 3 \times 10^{19} \mathrm{~cm}^{-3}$, which is twice larger than the $\mathrm{Be}$ concentration. This result suggests that the Be dopant also acts as a double donor in parent InAs without Fe.

Figures 9(a) and 9(c) show the band dispersion along the $\Gamma-\mathrm{K}-\mathrm{X}$ symmetry line taken with $p$ and $s$ polarizations, respectively. As shown in Fig. 9(a) taken with $p$ polarization, the holelike band dispersions centered at the $\Gamma$ point are the $\mathrm{LH}$ and SO bands, and the electronlike one near $E_{\mathrm{F}}$ centered at $\Gamma$ is the conduction band. As shown in Fig. 9(c), the SX-ARPES spectrum taken with the $s$ polarization shows only the $\mathrm{HH}$ band due to the matrix element effects. The band dispersions along the $\Gamma$-K-X symmetry line, as shown in Figs. 9(b) and 9(d), indicate that the EP can be seen only in the spectrum taken with $p$ polarization. The polarization dependence observed in InAs:Be is the same as that in (In,Fe)As:Be. Because the carrier concentration of the InAs:Be thin film is higher than that of the (In,Fe)As:Be one, all the band dispersions of the InAs:Be are shifted downward compared with those of the (In,Fe)As:Be.

\section{APPENDIX F: SX-ARPES SPECTRA OF PARAMAGNETIC (In,Fe)As}

The paramagnetic (In,Fe)As thin film without Be doping has a low electron-carrier concentration below the threshold 

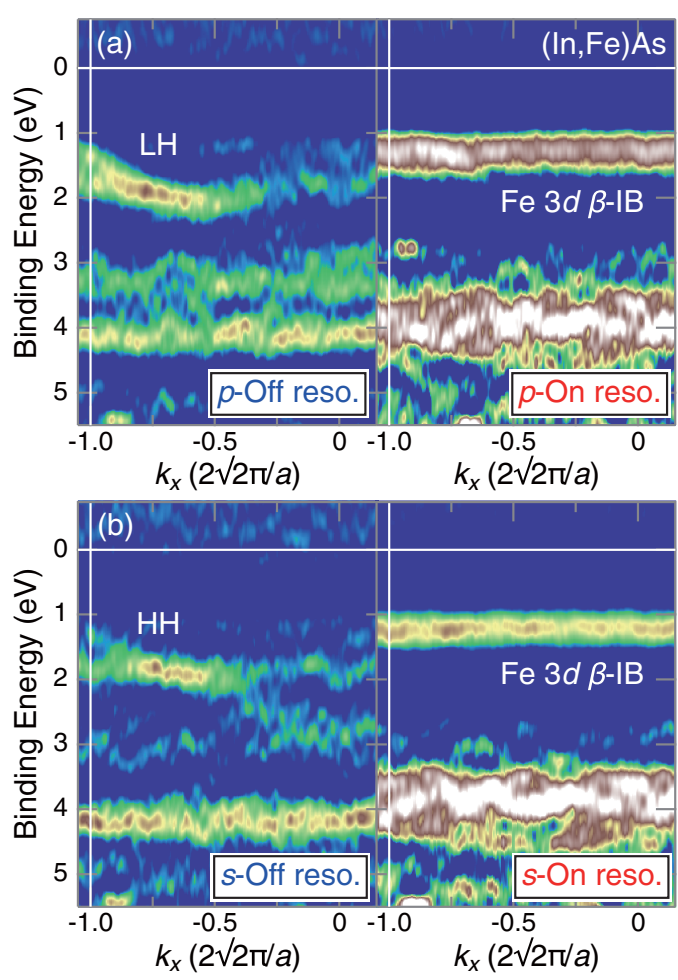

FIG. 11. Fe $L_{3}$ resonant ARPES spectra of paramagnetic $(\mathrm{In}, \mathrm{Fe}) \mathrm{As}$. (a, b) rARPES spectra taken with $p$ and $s$ polarizations, respectively. The left and right panels are the off- and on-resonance spectra, respectively. IB denotes the impurity band.

to induce ferromagnetism. Figure 10 shows the band dispersion of paramagnetic ( $\mathrm{In}, \mathrm{Fe})$ As. There is no intensity near $E_{\mathrm{F}}$ at the $\Gamma$ point. Additionally, all the band dispersions shift toward $E_{\mathrm{F}}$ compared with those of the Be-doped films, consistent with the low carrier concentration of the paramagnetic $(\mathrm{In}, \mathrm{Fe}) \mathrm{As}$. The quality of the data is lower than those of the (In,Fe)As:Be and InAs:Be thin films, because the thickness of the amorphous As capping layer on the (In,Fe)As thin film is thicker, and the thick capping layer weakens the ARPES signals. As shown in Figs. 3(a) and 3(c) (see the edges of VB and/or CB in the energy distribution curves, EDCs, at $\Gamma$ ), the VBM of (In,Fe)As is located around $E_{B} \sim 0.6 \mathrm{eV}$, indicating that the band gap is larger than $\sim 0.6$ $\mathrm{eV}$, while the band gap of (In,Fe)As:Be estimated from the energy difference between the VBM and CBM is $\sim 0.54 \mathrm{eV}$. This result indicates that the band gap of (In,Fe)As:Be shrunk compared to that of (In,Fe)As. Similar shrinking of the band gap observed in $\mathrm{K}_{3} \mathrm{C}_{60}$ compared to $\mathrm{C}_{60}$ has been explained as an effect beyond the one-electron potential approximation [43]. In contrast to insulating $\mathrm{C}_{60}$, a conduction electron is well screened by carriers in metallic $\mathrm{K}_{3} \mathrm{C}_{60}$. This leads to the decrease of the band gap with increasing the carrier concentration. Thus it is likely that the band gap of conducting $(\mathrm{In}, \mathrm{Fe}) \mathrm{As}$ :Be smaller than that of semiconducting (In,Fe)As originates from the same effect.

rARPES enables us to identify the Fe-3 $d$ IB states in (In,Fe)As. Figure 11 shows the $\mathrm{Fe} L_{3}$ rARPES spectra of the paramagnetic $(\mathrm{In}, \mathrm{Fe}) \mathrm{As}$ thin film taken with $p$ and $s$ polarizations. It should be noted that there is no Fe-3d $\alpha$-IB near $E_{\mathrm{F}}$, although the $\mathrm{Fe}-3 d \beta$-IB appears in the valence band at the resonance. Considering the observations of $(\mathrm{In}, \mathrm{Fe}) \mathrm{As}$ : $\mathrm{Be}$, the results indicate that the appearance of the $\mathrm{Fe}-3 d \alpha$-IB located near the conduction-band minimum depends on the carrier concentration, as discussed in the main text.
[1] H. Ohno, Science 281, 951 (1998).

[2] S. A. Wolf, D. D. Awschalom, R. A. Buhrman, J. M. Daughton, S. von Molnár, M. L. Roukes, A. Y. Chtchelkanova, and D. M. Treger, Science 294, 1488 (2001).

[3] I. Žutić, A. Matos-Abiague, B. Scharf, H. Dery, and K. Belashchenko, Mater. Today 22, 85 (2019).

[4] T. Dietl, Nat. Mater. 9, 965 (2010).

[5] M. Tanaka, S. Ohya, and P. N. Hai, Appl. Phys. Rev. 1, 011102 (2014).

[6] T. Nakamura, L. D. Anh, Y. Hashimoto, S. Ohya, M. Tanaka, and S. Katsumoto, Phys. Rev. Lett. 122, 107001 (2019).

[7] K. Takiguchi, L. D. Anh, T. Chiba, T. Koyama, D. Chiba, and M. Tanaka, Nat. Phys. 15, 1134 (2019).

[8] N. H. D. Khang and P. N. Hai, J. Appl. Phys. 126, 233903 (2019)

[9] N. T. Tu, P. N. Hai, L. D. Anh, and M. Tanaka, Appl. Phys. Lett. 108, 192401 (2016).

[10] A. V. Kudrin, Yu. A. Danilov1, V. P. Lesnikov, M. V. Dorokhin, O. V. Vikhrova, D. A. Pavlov, Yu. V. Usov, I. N. Antonov, R. N. Kriukov, A. V. Alaferdov, and N. A. Sobolev, J. Appl. Phys. 122, 183901 (2017).

[11] L. D. Anh, P. N. Hai, Y. Kasahara, Y. Iwasa, and M. Tanaka, Phys. Rev. B 92, 161201(R) (2015).
[12] L. D. Anh, P. N. Hai, and M. Tanaka, Nat. Commun. 7, 13810 (2016).

[13] P. N. Hai, L. D. Anh, S. Mohan, T. Tamegai, M. Kodzuka, T. Ohkubo, K. Hono, and M. Tanaka, Appl. Phys. Lett. 101, 182403 (2012).

[14] P. N. Hai, L. D. Anh, and M. Tanaka, Appl. Phys. Lett. 101, 252410 (2012).

[15] N. D. Vu, T. Fukushima, K. Sato, and H. Katayama-Hoshida, Jpn. J. Appl. Phys. 53, 110307 (2014).

[16] T. Dietl, H. Ohno, and F. Matsukura, Phys. Rev. B 63, 195205 (2001).

[17] M. Kobayashi, I. Muneta, Y. Takeda, Y. Harada, A. Fujimori, J. Krempasky, T. Schmitt, S. Ohya, M. Tanaka, M. Oshima, and V. N. Strocov, Phys. Rev. B 89, 205204 (2014).

[18] J. Krempasky, S. Muff, F. Bisti, M. Fanciulli, H. Volfová, A.P. Weber, N. Pilet, P. Warnicke, H. Ebert, J Braun, F. Bertran, V. V. Volobuev, J. Minár, G. Springholz, J. H. Dil, and V. N. Strocov, Nat. Commun. 7, 13071 (2016).

[19] S. Sakamoto, Y. K. Wakabayashi, Y. Takeda, S.-i. Fujimori, H. Suzuki, Y. Ban, H. Yamagami, M. Tanaka, S. Ohya, and A. Fujimori, Phys. Rev. B 95, 075203 (2017).

[20] T. Takeda, M. Suzuki, L. D. Anh, N. T. Tu, T. Schmitt, S. Yoshida, M. Sakano, K. Ishizaka, Y. Takeda, S.-I. Fujimori, 
M. Seki, H. Tabata, A. Fujimori, V. N. Strocov, M. Tanaka, and M. Kobayashi, Phys. Rev. B 101, 155142 (2020).

[21] N. J. Kawai, T. Nakagawa, T. Kojima, K. Ohta, and M. Kawashima, Electron. Lett. 20, 47 (1984).

[22] V. N. Strocov, L. L. Lev, M. Kobayashi, C. Cancellieri, M.-A. Husanua, A. Chikina, N. B. M. Schröter, X. Wang, J. A. Krieger, and Z. Salman, J. Electron Spectrosc. Relat. Phenom. 236, 1 (2019).

[23] V. N. Strocov, X. Wang, M. Shi, M. Kobayashi, J. Krempasky, C. Hess, T. Schmitt, and L. Patthey, J. Synchrotron Radiat. 21, 32 (2014).

[24] V. N. Strocov, T. Schmitt, U. Flechsing, T. Schmidt, A. Imhof, Q. Chen, J. Raabe, R. Betemps, D. Zimoch, J. Krempasky, X. Wang, M. Grioni, A. Piazzalunga, and L. Patthey, J. Synchrotron Rad. 17, 631 (2010).

[25] G. K. Wertheim, J. E. Rowe, D. N. E. Buchanan, E. E. Chaban, A. F. Hebard, A. R. Kortan, A. V. Makhija, and R. C. Haddon, Science 252, 1419 (1991).

[26] J. H. Weaver, P. J. Benning, F. Stepniak, and D. M. Poirier, J. Phys. Chem. Solids 53, 1707 (1992).

[27] M. Kobayashi, L. D. Anh, P. N. Hai, Y. Takeda, S. Sakamoto, T. Kadono, T. Okane, Y. Saitoh, H. Yamagami, Y. Harada, M. Oshima, M. Tanaka, and A. Fujimori, Appl. Phys. Lett. 105, 032403 (2014).

[28] P. W. Anderson, Phys. Rev. 124, 41 (1964).

[29] J. R. Schrieffer and P. A. Wolff, Phys. Rev. 149, 491 (1966).

[30] J. Okabayashi, A. Kimura, O. Rader, T. Mizokawa, A. Fujimori, T. Hayashi, and M. Tanaka, Phys. Rev. B 58, R4211(R) (1998).

[31] P. Manca, J. Phys. Chem. Solids 20, 268 (1961).

[32] B. Gu and S. Maekawa, Phys. Rev. B 94, 155202 (2016).

[33] K. Sato, L. Bergqvist, J. Kudrnovský, P. H. Dederichs, O. Eriksson, I. Turek, B. Sanyal, G. Bouzerar, H. Katayama-
Yoshida, V. A. Dinh, T. Fukushima, H. Kizaki, and R. Zeller, Rev. Mod. Phys. 82, 1633 (2010).

[34] K. Zhao, Z. Deng, X. C. Wang, W. Han, J. L. Zhu, X. Li, Q. Q. Liu, R. C. Yu, T. Goko, B. Frandsen, Lian Liu, Fanlong Ning, Y. J. Uemura, H. Dabkowska, G. M. Luke, H. Luetkens, E. Morenzoni, S. R. Dunsiger, A. Senyshyn, P. Böni, and C. Q. Jin, Nat. Commun. 4, 1442 (2013).

[35] F. C. Zhang and T. M. Rice, Phys. Rev. B 37, 3759 (1988).

[36] K. Tsutsui, T. Tohyama, and S. Maekawa, Phys. Rev. Lett. 83, 3705 (1999).

[37] A. Damascelli, Z. Hussain, and Z.-X. Shen, Rev. Mod. Phys. 75, 473 (2003).

[38] M. Kobayashi, I. Muneta, T. Schmitt, L. Patthey, S. Ohya, M. Tanaka, M. Oshima, and V. N. Strocov, Appl. Phys. Lett. 101, 242103 (2012).

[39] T. Kataoka, M. Kobayashi, G. S. Song, Y. Sakamoto, A. Fujimori, F.-H. Chang, H.-J. Lin, D. J. Huang, C. T. Chen, S. K. Mandal, T. K. Nath, D. Karmakar, and I. Dasgupta, Jpn. J. Appl. Phys. 48, 04C200 (2009).

[40] C. T. Chen, Y. U. Idzerda, H.-J. Lin, N. V. Smith, G. Meigs, E. Chaban, G. H. Ho, E. Pellegrin, and F. Sette, Phys. Rev. Lett. 75, 152 (1995).

[41] W. L. Yang, A. P. Sorini, C-C. Chen, B. Moritz, W.-S. Lee, F. Vernay, P. Olalde-Velasco, J. D. Denlinger, B. Delley, J.-H. Chu, J. G. Analytis, I. R. Fisher, Z. A. Ren, J. Yang, W. Lu, Z. X. Zhao, J. van den Brink, Z. Hussain, Z.-X. Shen, and T. P. Devereaux, Phys. Rev. B 80, 014508 (2009).

[42] E. Engel and S. H. Vosko, Phys. Rev. B 50, 10498 (1994).

[43] T. Takahashi, S. Suzuki, T. Morikawa, H. Katayama-Yoshida, S. Hasegawa, H. Inokuchi, K. Seki, K. Kikuchi, S. Suzuki, K. Ikemoto, and Y. Achiba, Phys. Rev. Lett. 68, 1232 (1992). 